\title{
THC exposure of human iPSC neurons impacts genes associated with neuropsychiatric disorders
}

\author{
Boris Guennewig ${ }^{1,2,3}$, Maina Bitar ${ }^{4}$, Ifeanyi Obiorah ${ }^{5,6}$, James Hanks ${ }^{5,7,8}$, Elizabeth A. O'Brien ${ }^{4}$, Dominik C. Kaczorowski ${ }^{3}$, \\ Yasmin L. Hurd ${ }^{5,9}$, Panos Roussos 5,7, , Kristen J. Brennand (D, ${ }^{5,6}$ and Guy Barry ${ }^{4}$
}

\begin{abstract}
There is a strong association between cannabis use and schizophrenia but the underlying cellular links are poorly understood. Neurons derived from human-induced pluripotent stem cells (hiPSCs) offer a platform for investigating both baseline and dynamic changes in human neural cells. Here, we exposed neurons derived from hiPSCs to $\Delta^{9}-$ tetrahydrocannabinol (THC), and identified diagnosis-specific differences not detectable in vehicle-controls. RNA transcriptomic analyses revealed that THC administration, either by acute or chronic exposure, dampened the neuronal transcriptional response following potassium chloride (KCl)-induced neuronal depolarization. THC-treated neurons displayed significant synaptic, mitochondrial, and glutamate signaling alterations that may underlie their failure to activate appropriately; this blunted response resembles effects previously observed in schizophrenia hiPSCderived neurons. Furthermore, we show a significant alteration in THC-related genes associated with autism and intellectual disability, suggesting shared molecular pathways perturbed in neuropsychiatric disorders that are exacerbated by THC.
\end{abstract}

\section{Introduction}

Human-induced pluripotent stem cells (hiPSCs) serve as a tool for the study of developmental processes and disease-relevant models. This has been especially valuable for the study of the human brain where primary tissue for study has been the most difficult to obtain. hiPSCs have provided mechanistic insights into both neurodevelopmental disorders ${ }^{1}$ and neurodegenerative diseases ${ }^{2,3}$. Research into psychiatric disorders such as autism ${ }^{4}$, bipolar disease ${ }^{5}$, and schizophrenia ${ }^{6}$ have greatly benefited from the insights afforded by hiPSCs, as these are largely considered human- specific disorders. hiPSCbased models facilitate isogenic investigations into

\footnotetext{
Correspondence: Guy Barry (Guy.Barry@qimrberghofer.edu.au)

${ }^{1}$ Sydney Medical School, Brain and Mind Centre, The University of Sydney,

Camperdown, Sydney, NSW, Australia

${ }^{2}$ St. Vincent's Clinical School and School of Biotechnology and Biomolecular

Sciences, University of New South Wales, Kensington, NSW, Australia

Full list of author information is available at the end of the article

These authors contributed equally: Kristen J. Brennand and Guy Barry.
}

molecular and environmental factors that may exacerbate or ameliorate disease predisposition.

The widespread use of cannabis calls for a concerted effort into increased understanding of both the positive and negative effects of the drug. Brain imaging studies of the primary psychoactive component of cannabis, $\Delta^{9}$-tetrahydrocannabinol (THC), demonstrated structural and functional changes following regular cannabis use ${ }^{7}$, while molecular studies uncovered signaling pathways downstream of the two cannabinoid (CB) receptors, CB1, and CB2. Depression of glutamate signaling is a common feature of THC-induced effects via the CB1 receptor in both humans and in animal models ${ }^{8,9}$.

There is a significant association between cannabis use and schizophrenia in human subjects ${ }^{10-14}$, however, whether this reflects patient self-medication of prodromal symptoms or an environmental modulation of genetic susceptibility remains an ongoing discussion ${ }^{15,16}$. We recently reported molecular abnormalities in 
schizophrenia patient hiPSC-derived neurons in response to neural activity ${ }^{6}$; here we describe a distinct overlap in hypo-excitability, particularly in the glutamate system, between schizophrenia patient-derived neurons and those treated with THC. THC exposure seems to deregulate glutamate receptors and other genes involved in synaptic function. We observe significant THC-dependent changes in postsynaptic density, ion channel and WNT pathway genes, and epigenetic regulators; and molecular connections to autism and intellectual disability. Although the molecular mechanisms may not be precisely the same, the convergence of glutamatergic hypo-function may partially explain the increased risk for psychiatric disorders amongst those exposed to cannabis.

\section{Materials and methods}

\section{Generation of hiPSC neurons and RNA sequencing}

Human fibroblasts were obtained from ATCC (CRL2522) and Coriell (control: GM03440, GM03651, GM04506, AG09319, AG09429; SZ: GM01792, GM02038, GM01835, and GM02497). Limited phenotypic information for each donor is available from the Coriell Cell Repository, and summarized in the methods of Topol et al ${ }^{17}$. Unfortunately, THC exposure status for each donor is unknown. hiPSCs were reprogrammed using tetracycline-inducible lentiviral vectors and differentiated to neural precursor cells (NPCs) as previously described $^{18}$. NPCs were differentiated on poly- ornithine/ laminin coated plates for 6 weeks. Passage-matched NPCs were used for all experiments. All hiPSC and NPCs used were mycoplasma-free. Forebrain neural progenitor cells were generated from five control and four case hiPSCs as previously reported ${ }^{6,18,19}$ and neurons were differentiated according to a 6-week maturation protocol. Samples used in RNA sequencing or quantitative RT-PCR can be found in Supplementary Table S1. THC was dissolved in DMSO to $1 \mathrm{mg} / \mathrm{ml}$ and prepared as previously described; ${ }^{20}$ in all experiments, an equivalent volume of DMSO was used as a vehicle control. Acute $(1 \mu \mathrm{M}$ THC for $24 \mathrm{~h})$ and chronic (50 nM THC; five treatments over 7 days) THC exposure (and DMSO-vehicle control) occurred immediately prior to collection. $\mathrm{KCl}$ was dissolved in $\mathrm{PBS}$ as previously described $^{6}$; in all experiments, an equivalent volume of PBS was used as a vehicle control. $50 \mathrm{mM} \mathrm{KCl}$ treatment occurred for the final three hours prior to collection; consistent with our previous molecular ${ }^{6}$ and neurotransmitter release ${ }^{21}$ studies. For RNA-seq experiments, two wells per individual were treated. The RNA Integrity Number (RIN) was determined using an RNA Nano chip (Agilent Technologies) on the Agilent 2100 Bioanalyzer. All samples have high RIN (mean \pm SD: $9.54 \pm 0.21$ ). $500 \mathrm{ng}$ of total RNA was used as input material for library preparation using the TruSeq Stranded Total RNA Kit (Illumina, USA).

\section{Processing of RNA sequencing data and analyses}

RNA sequencing data has been deposited into Sequence Read Archive (SRA; PRNJA419702, "RNA-Seq of iPSCderived neurons"). Reads were mapped to GRCh38.p5 reference genome using STAR (version 2.5.1a). Known Gencode gene levels (version 24) were quantified by RSEM (version 1.3.0). To facilitate inter-dataset comparisons, we performed ranked (Spearman) and unranked correlation (Pearson) analysis of the controls in both the $\pm \mathrm{KCl}$ and $\pm \mathrm{THC}$ datasets, and confirmed that the samples are highly comparable (all control comparisons are $\geq$ 97\%). Differentially expressed genes were identified with edge $\mathrm{R}$ in $\mathrm{R}$ after TMM normalization and filtering. $p$-values and false discovery rate (FDR) were calculated and differentially expressed genes (DEG) were determined as those with an estimated $p$-value $\leq 0.05$ and $\mathrm{FDR} \leq 0.01$.

\section{Gene sets for enrichment analyses}

To further characterize the DEGs we performed enrichment analysis, using a group of gene sets for known molecular pathways and biological processes, including: Gene Ontology (GO) sets of molecular functions (MF), biological processes (BP), and cellular components (CC) (http://www.geneontology.org); the KEGG dataset (http:// www.genome.jp/kegg/pathway.html); and the HUGO Gene Nomenclature Committee (HGNC) gene families (http://www.genenames.org). The genes in each gene set were tested for overlap using Fisher's exact test and FDR correction. Differential expressed genes were (i) separated in upregulated and downregulated genes; (ii) analyzed for full GO overrepresentation according to hypergeometric testing with a significance cutoff FDR $=0.05$ in BiNGO (version 3.0.3); (iii) processed with the enrichment map pipeline (https://f1000research.com/posters/5-1235) a $p$-value cutoff $=0.001, q$-value cutoff $=0.05$ and Jaccard coefficient cutoff $=0.25$ and (iv) visualized in Cytoscape (version 3.5.1).

\section{Quantitative RT-PCR}

For qPCR experiments, three wells per individual were treated with either DMSO- vehicle control for 7 days, acute THC exposure $(1 \mu \mathrm{M}$ THC for $24 \mathrm{~h})$ or chronic THC treatment (five treatments with $50 \mathrm{nM}$ THC over 7 days) immediately prior to collection at 6 weeks. Candidate genes were validated for THC-treated and activitytreated alterations using quantitative RT-PCR. cDNA synthesis was performed using the SuperScript III FirstStrand Synthesis System (ThermoFisher Scientific, USA). Briefly, $500 \mathrm{ng}$ of total RNA was used and random hexamer primed protocol was followed. Each cDNA sample was amplified in triplicate using SYBR Green PCR Master Mix (ThermoFisher Scientific, USA). Primer pairs used for this analysis are described in Supplementary Table S2. 


\section{Generation of gene datasets}

As no up to date datasets for associated genes were available for autism, intellectual disability or schizophrenia, we generated our own through extensive literature and database searches. Specific details are available in the Supplemental Information 'Generation of Gene Databases'.

\section{Results}

\section{hiPSC-derived neurons as a model for THC biology}

To gain further insight into THC-related molecular mechanisms we utilized hiPSC-derived neurons from four controls as previously reported ${ }^{6}$. THC (or vehicle control) was added to hiPSC-derived neurons from each individual as acute $(1 \mu \mathrm{M}$ THC for $24 \mathrm{~h})$ or chronic $(50 \mathrm{nM} \mathrm{THC}$; five treatments over 7 days) treatments. Acute and chronic THC concentrations were rationally selected from studies of primary mouse neurons ${ }^{22}$ and experimentally validated in hiPSC neurons ${ }^{23}$. RNA was extracted and subjected to RNA sequencing (RNA-seq) using the Illumina platform. Our bioinformatic analyses pipeline combined integrated genome/transcriptome alignment using STAR, quantification using RSEM and differential expression using EdgeR. Relative to vehicle treatment, acute THC exposure resulted in 497 genes significantly altered in hiPSC- derived neurons compared to untreated controls, while chronic THC exposure perturbed 810 genes (Fig. 1a; Supplementary Table S3; Supplementary Figure 1). The overlap between acute and chronic exposures was highly significant (421 genes; $p$ value $=0 \mathrm{e}+00$, odds ratio $=586.5$, Fisher's exact test). Specific subsets of genes involved in the glutamate receptor pathway and mitochondrial function were altered in response to acute or chronic THC exposure (Supplementary Table S4; Supplementary Figure 2; Fig. $1 \mathrm{~b}-\mathrm{d}$ ) and have previously been implicated in THC biology ${ }^{8,24,25}$. These results provide data to support the use of hiPSC-derived neurons as a model for investigating THC responses in an in vitro human neuronal system.

\section{RNA sequencing implicates synaptic function,}

\section{demethylation and ion channel function in THC-treated} hiPSC neurons

Closer inspection of functional gene clusters associated with THC treatment revealed the potential contribution of genes involved at the postsynaptic density such as HOMER1, GRID2, GRIK1, and SIPA1L1 (Fig. 2a, b). Moreover, chronic THC treatments resulted in the alteration of additional synaptic related genes such as SYNGAP1 and SHANK1. Ion channel genes, especially potassium voltage-gated channel genes (KCNE4, KCNA4, $K C N J 10$, and $K C N N 3$ ) are also responsive to both THC treatments with further ion channel genes (KCNJ2, KCNA2, and KCNT2) associated following chronic THC exposure (Fig. 2a). These results strongly implicate synaptic function as a key target of THC-mediated responses. Interestingly, we found epigenetic related transcriptional responses evident in both acute and chronic THC exposures that included the alterations of genes involved in the dynamic methylation/demethylation process (DNMT1, GADD45B, and $A P O B E C 3 C$ ); chronic THC exposure resulted in further decreases of histone modification-related proteins such as SETD1A, SETD5, CBX6, KMT2A, KMT2C, and NCOA6 and methyl binding proteins $M E C P 2$ and $M B D 5$ (Supplementary Table S5). Network analyses (Enrichment map pipeline in Cytoscape) using the genes altered in response to THC exposure reinforce the involvement of pathways linked to developmental, chromatin regulation and mitochondrial biology (Fig. 2c; Supplementary Table S6).

\section{THC exposure significantly alters genes implicated in autism and intellectual disability}

We noticed that many genes implicated in psychiatric disease coincided with genes altered in response to THC treatments. In order to calculate statistical relevance we needed to first update the numbers of genes associated with these disorders and found genes related to autism spectrum disorder (1037 genes), intellectual disability (2461 genes) and schizophrenia (723 genes; see Supplementary Information 'Generation of Gene Databases' for details; Supplementary Table S7). Included in our list of significantly altered transcripts following THC exposure is a substantial number of genes linked to autism (80 genes) and intellectual disability (167 genes), with fewer overlapping with schizophrenia (Fig. 3a); autism and intellectual disability associated genes are significant for both $p$ value and odds ratio using the Fisher's exact test (Fig. 3b). These data suggest that endogenous THC responsive pathways include many psychiatric disease-associated genes and that changes in these genes, either genetically or epigenetically, may contribute to cannabis-related adverse reactions such as psychosis in some users.

\section{Overlapping signaling pathways between THC and schizophrenia}

We compared the bioinformatic results from our current THC studies to data obtained from our previous studies of schizophrenia patient hiPSC-derived neurons $^{6,18}$ to ensure that the quality of the differentiations were comparable across experiments (Supplementary Figure 3). Raw data from all subjects from Roussos et $\mathrm{al}^{6}$. was applied to our bioinformatic pipeline. WNT and mitochondrial pathways (Supplementary Table S8) were significantly altered in both our current THC and previous schizophrenia studies ${ }^{18,26}$. Genes related to ion channel function were also highly represented in both the THC and schizophrenia gene lists (Supplementary Table 


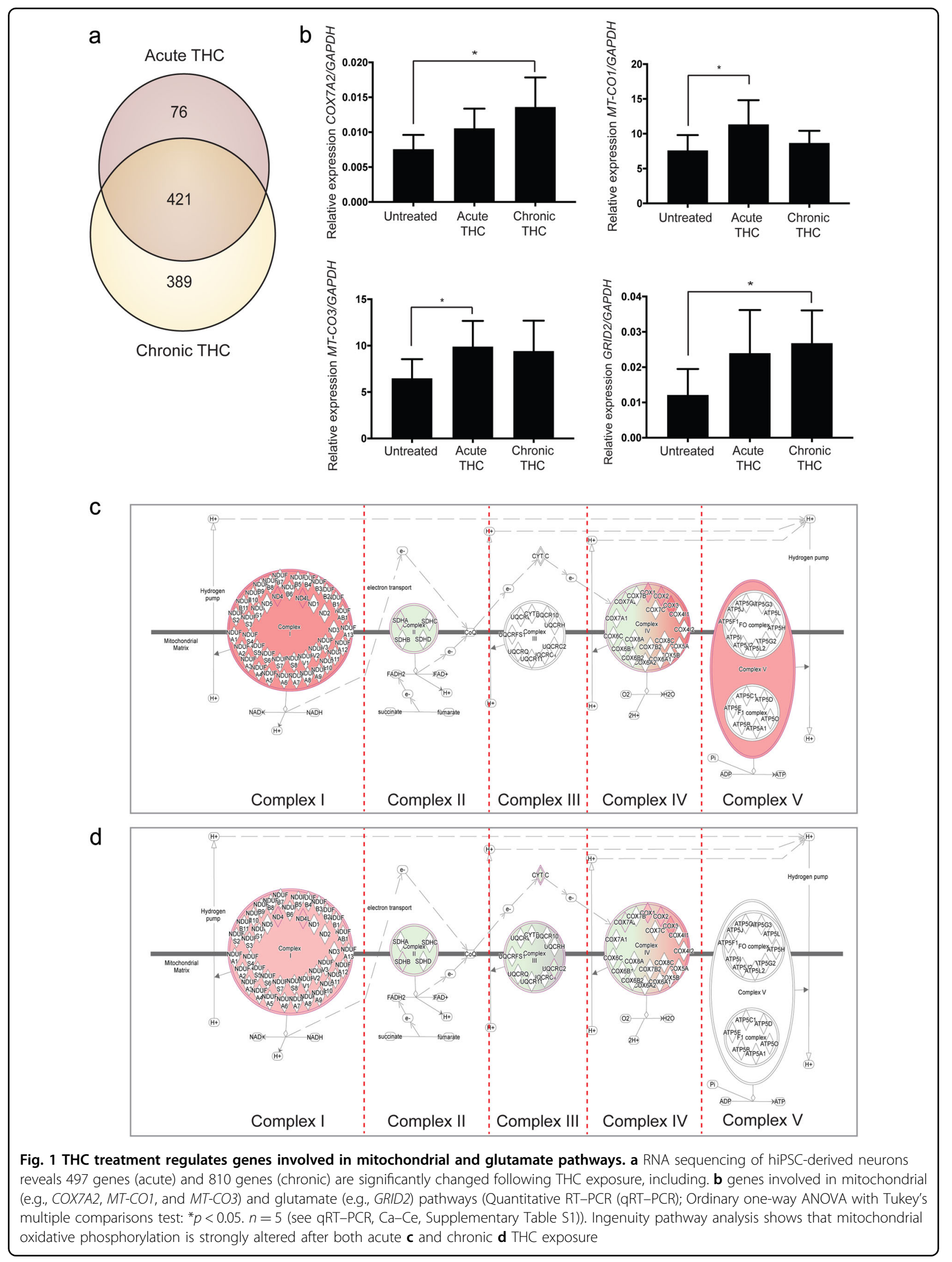




\begin{tabular}{|c|c|c|c|c|}
\hline \multirow[b]{2}{*}{ Postsynaptic density } & \multicolumn{2}{|c|}{ Acute THC } & \multicolumn{2}{|c|}{ Chronic THC } \\
\hline & $\begin{array}{l}\text { Fold } \\
\text { change }\end{array}$ & P-value & $\begin{array}{l}\text { Fold } \\
\text { change }\end{array}$ & $P$-value \\
\hline GRID2 & 2.08 & 0.00013 & 2.26 & 3.22E-05 \\
\hline CAP2 & 1.73 & $8.91 \mathrm{E}-06$ & 1.44 & 0.00020 \\
\hline GRIK1 & 1.41 & 4.76E-05 & 1.62 & 2.67E-06 \\
\hline SIPA1L1 & 1.27 & 5.59E-05 & 1.32 & 2.81E-05 \\
\hline HOMER1 & -2.46 & $1.45 \mathrm{E}-07$ & -2.56 & 4.66E-08 \\
\hline SYNPO & - & $\mathrm{n} / \mathrm{s}$ & 2.34 & 0.00015 \\
\hline SYNGAP1 & - & $\mathrm{n} / \mathrm{s}$ & 2.14 & 0.00027 \\
\hline PTCH1 & - & $\mathrm{n} / \mathrm{s}$ & 2.14 & 0.00016 \\
\hline NTRK2 & - & $n / s$ & 2.09 & 1.64E-05 \\
\hline sos1 & - & $\mathrm{n} / \mathrm{s}$ & 1.80 & 0.00032 \\
\hline RUSC1 & - & $\mathrm{n} / \mathrm{s}$ & 1.56 & 0.00023 \\
\hline SRGAP2 & - & $n / s$ & 1.30 & 0.00014 \\
\hline GSK3B & - & $\mathrm{n} / \mathrm{s}$ & 1.27 & 0.00015 \\
\hline EPHA4 & - & $\mathrm{n} / \mathrm{s}$ & -1.56 & 0.00028 \\
\hline SHANK1 & - & $n / s$ & -4.39 & 0.00026 \\
\hline Ion channel & $\begin{array}{l}\text { Fold } \\
\text { change }\end{array}$ & P-value & $\begin{array}{c}\text { Fold } \\
\text { change }\end{array}$ & P-value \\
\hline TMEM38A & -2.04 & $3.14 \mathrm{E}-05$ & - & $\mathrm{n} / \mathrm{s}$ \\
\hline KCNF1 & -2.87 & 7.98E-05 & - & $\mathrm{n} / \mathrm{s}$ \\
\hline KCNK15 & -8.86 & $8.45 \mathrm{E}-05$ & - & $\mathrm{n} / \mathrm{s}$ \\
\hline RYR3 & 2.58 & $5.69 \mathrm{E}-05$ & 2.59 & 5.17E-05 \\
\hline GRID2 & 2.08 & 0.00013 & 2.26 & 3.22E-05 \\
\hline GRIK1 & 1.41 & 4.76E-05 & 1.62 & $2.67 \mathrm{E}-06$ \\
\hline CACNG7 & 1.89 & 4.45E-05 & 2.10 & 4.55E-06 \\
\hline KCNJ10 & 1.74 & $1.05 \mathrm{E}-05$ & 2.05 & 1.62E-07 \\
\hline KCNN3 & 2.48 & 1.15E-05 & 2.27 & $5.82 \mathrm{E}-05$ \\
\hline ITPR2 & 1.18 & $4.24 \mathrm{E}-05$ & 1.21 & $2.38 \mathrm{E}-05$ \\
\hline LRRC8E & -2.92 & $7.83 \mathrm{E}-07$ & -2.40 & 2.39E-05 \\
\hline KCNA4 & $\begin{array}{l}-3.99 \\
\end{array}$ & $9.22 \mathrm{E}-06$ & -3.59 & $5.23 E-05$ \\
\hline KCNE4 & -5.42 & $5.31 \mathrm{E}-08$ & -4.55 & 2.83E-06 \\
\hline FXYD6 & - & $\mathrm{n} / \mathrm{s}$ & 2.17 & 0.00025 \\
\hline ASIC1 & - & $n / s$ & 1.82 & 0.00011 \\
\hline TMEM63A & - & $n / s$ & 1.45 & 0.00016 \\
\hline KCNT2 & - & $\mathrm{n} / \mathrm{s}$ & 1.44 & 0.00019 \\
\hline KCNJ2 & - & $\mathrm{n} / \mathrm{s}$ & -1.67 & 0.00022 \\
\hline CHRNA9 & - & $n / s$ & -2.88 & 0.00026 \\
\hline KCNQ1 & - & $n / s$ & -6.47 & 0.00016 \\
\hline KCNK6 & - & $\mathrm{n} / \mathrm{s}$ & -6.81 & $9.78 \mathrm{E}-05$ \\
\hline
\end{tabular}
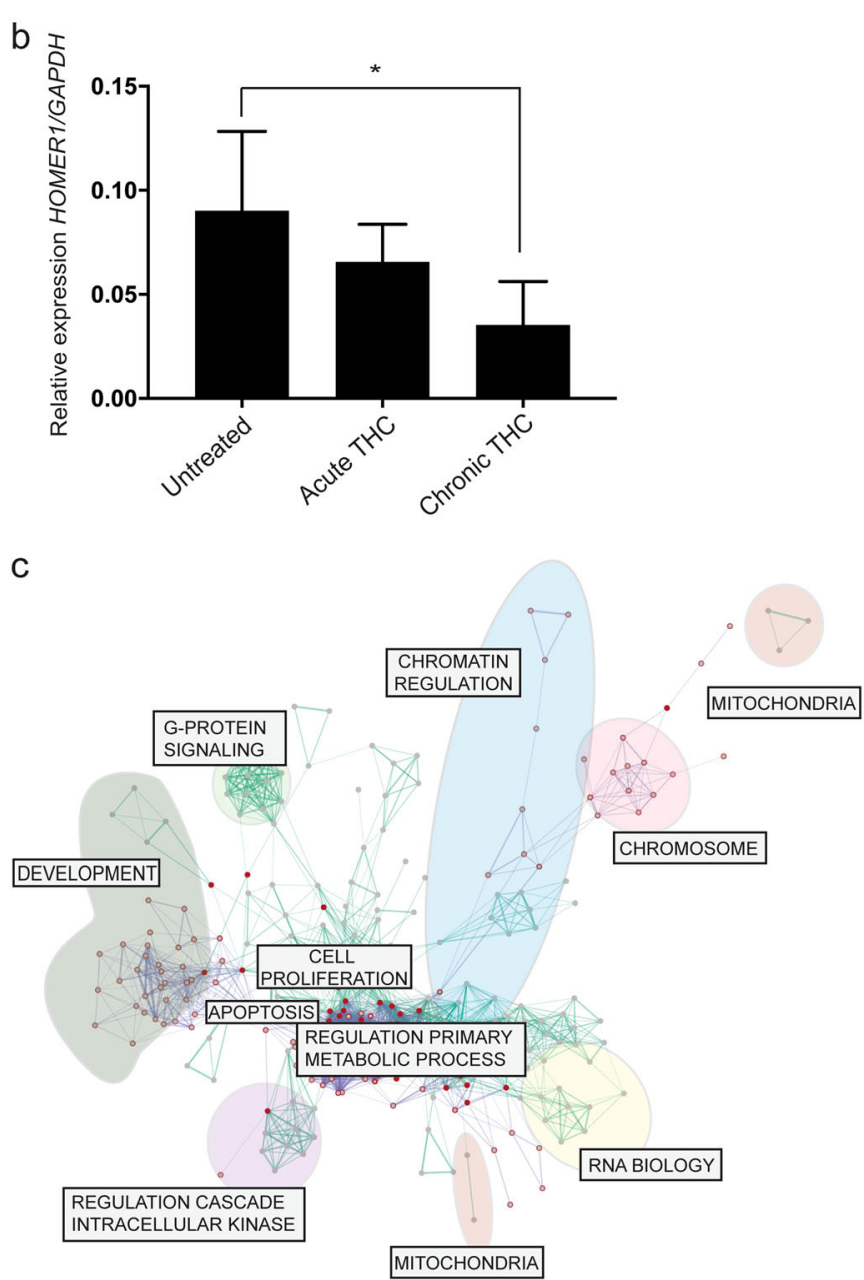

Fig. 2 Postsynaptic density and ion channel genes are regulated by THC treatment. a, b Multiple postsynaptic density and ion channel genes are significantly altered in hiPSC-derived neurons following acute or chronic THC exposure, including the postsynaptic gene HOMER1 (Quantitative RT-PCR (qRT-PCR); Ordinary one-way ANOVA with Tukey's multiple comparisons test: * $p<0.05 . n=5$ (see qRT-PCR, Ca-Ce, Supplementary Table S1)). c Network analysis combining all THC-related genes from acute and chronic THC treatment shows broad changes to fundamental cellular functions such as RNA biology, chromatin regulation and development

S8). Although these pathways were conserved, specific genes related to altered ion channel, WNT or mitochondrial function did not overlap.

\section{Blunted activity-dependent transcriptional response shared between THC and schizophrenia}

In our previous study ${ }^{6}$, we demonstrated that schizophrenia-associated hiPSC-derived neurons had a blunted transcriptomic response to $\mathrm{KCl}$ relative to controls. We repeated this experimental design on control hiPSC-derived neurons from four individuals, providing either acute $(1 \mu \mathrm{M}$ THC, $24 \mathrm{~h})$, chronic $(50 \mathrm{nM}$ THC, 7 days) or vehicle treatment, after which cells were activated using $50 \mathrm{mM} \mathrm{KCl}$ (or vehicle) for $3 \mathrm{~h}$ as before. We saw a significantly blunted transcriptomic response, more prominent with the acute $(75 \%$ reduction compared to
$\mathrm{KCl}$-activated control neurons; $p$-value $=1.3 \mathrm{e}-73$, odds ratio $=278.6$, Fisher's exact test) than the chronic exposure $(60 \%$ reduction compared to $\mathrm{KCl}$-activated control neurons; $p$-value $=4.4 \mathrm{e}-83$, odds ratio $=181.6$, Fisher's exact test) of THC (Fig. 4a; Supplementary Table S9; Supplementary Figure 4).

After re-running the raw schizophrenia-associated data from activity-dependent experiments conducted in Roussos et $\mathrm{al}^{6}$., we again saw a dramatic reduction ( 93\%; $p$-value $=4.3 \mathrm{e}-27$, odds ratio $=605.7$, Fisher's exact test) in the schizophrenia-associated transcriptomic response (Fig. 4b; Supplementary Table S10). We tested candidate genes on a cohort of schizophrenia-associated hiPSCderived neurons (Fig. 4c) and found blunted expression profiles for COX7A2, GRID2 and HOMER1 (Fig. 4d) using quantitative PCR. Quantitative PCR further confirmed 


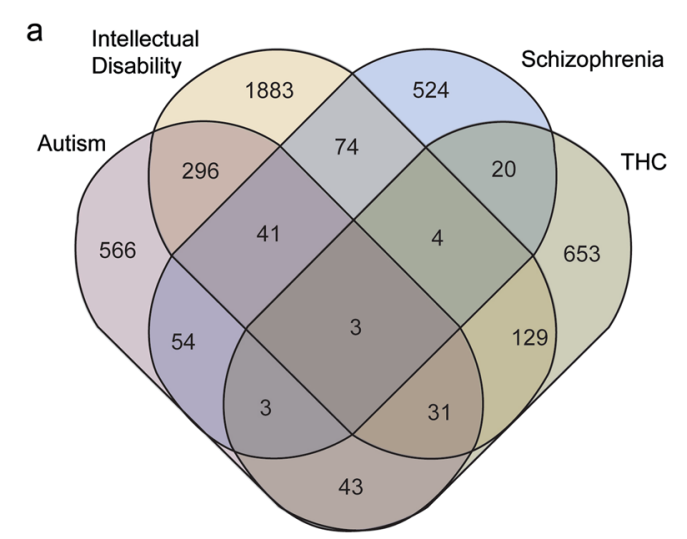

b
\begin{tabular}{|c|c|c|c|}
\hline & $\begin{array}{c}\text { Intellectual } \\
\text { disability }\end{array}$ & Autism & Schizophrenia \\
\hline Genes in dataset & 2461 & 1037 & 723 \\
\hline DEGs & 167 & 80 & 30 \\
\hline P-value & $1.10 \mathrm{E}-08$ & $1.10 \mathrm{E}-06$ & 0.68 \\
\hline Corrected P-value & $3.30 \mathrm{E}-08$ & $3.30 \mathrm{E}-06$ & 1 \\
\hline Odds ratio & 1.7 & 1.9 & 0.9 \\
\hline Jaccard index & 0.052 & 0.043 & 0.019 \\
\hline
\end{tabular}

Fig. 3 Genes altered by THC treatment in hiPSC-derived neurons are significantly associated with autism and intellectual disability. a Venn diagram showing the overlap between THC-related genes and autism, intellectual disability and schizophrenia. b THC-related genes are significantly related to autism and intellectual disability $(p$-value $<0.05)$

this blunting effect of THC exposure; significantly reduced expression of immediate early genes such as NR4A1 and FOSB was observed following $\mathrm{KCl}$ treatment (Fig. 4e, f), consistent with what we found previously for these genes in schizophrenia-associated hiPSC-derived neurons ${ }^{6}$.

\section{Discussion}

Our results show that the endogenous response to THC operates through molecular pathways that have been strongly associated with psychiatric disease. This implies that genetic and epigenetic variation present in these specific pathways in individuals might determine the extent of individual susceptibility to adverse response to THC. Genes involved in autism and intellectual disability are prominently involved in THC signaling, while schizophrenia risk may be more linked to similarities when activity- dependent pathways are disrupted.

Understanding human brain subtleties requires a manipulable neuronal model; here we validate hiPSCderived neuronal networks as a viable system for these types of studies. Others have similarly reported that treatment of hiPSC-derived dopaminergic neurons with THC reproduces effects observed in other mouse and human models ${ }^{27}$. Mitochondrial pathway dysfunction has been linked to THC exposure ${ }^{24,25,28-30}$ and schizophrenia ${ }^{26,31}$, a convergence that is captured in our system (Figs. 1b-d; 2c). Acute and chronic stress have differing effects on mitochondrial genes ${ }^{32}$ and the different respiratory chain complexes have unique functions during stress responses; ${ }^{33,34}$ this may explain the parallel differences between acute and chronic THC exposures observed here. Furthermore, links between the glutamatergic and ion channel pathways and THC reported here (Fig. 1b; 2a) are supported by previous studies where both glutamate and ion channel pathways are regulated via cannabinoid receptors ${ }^{8,35}$. Many of the ion channel proteins identified here are critical for acute synaptic activity $^{36}$ but are also involved in later stage neural proliferation and differentiation ${ }^{37,38}$, suggesting that THC exposure may exert short- and/or long-term effects in the developing human brain.

Our experimental system enables relevant comparisons to known disease-causing genes in humans and led us to the significant overlaps in THC-induced genes and those involved in autism and intellectual disability (Fig. 3b). Moreover, by studying activity-related changes instead of baseline differences, we detected similarities between THC-induced hypo-function and schizophrenia (Fig. 4a, b), which would have otherwise been missed in a static system or post-mortem tissue. This is important as we found no overlap of significantly altered ion channel, WNT or mitochondrial genes between THC and schizophrenia datasets (Supplementary Table S8), suggesting that THC- and schizophrenia-related signaling pathways are different with respect to specific genes but convergent in function.

To test whether there was a significant enrichment for psychiatric disease genes in our THC results, we first constructed a list of disease-related genes from the literature, as there were no complete and up to date collections available. We generated comprehensive lists of currently known genes implicated in schizophrenia, autism and intellectual disability, finding significant correlations between THC treatment and autism and intellectual disability (Fig. 3b; Supplementary Table S7). Interestingly, a large recent GWAS uncovered four genes that were significantly associated with lifetime cannabis use ${ }^{39}$ : one, KCNT2, showed significant THC-responsiveness in our hiPSC-derived neurons (Fig. 2a), while two others, NCAM1 and CADM2 (also known as SynCAM2) are important for postsynaptic function ${ }^{40,41}$, consistent with 


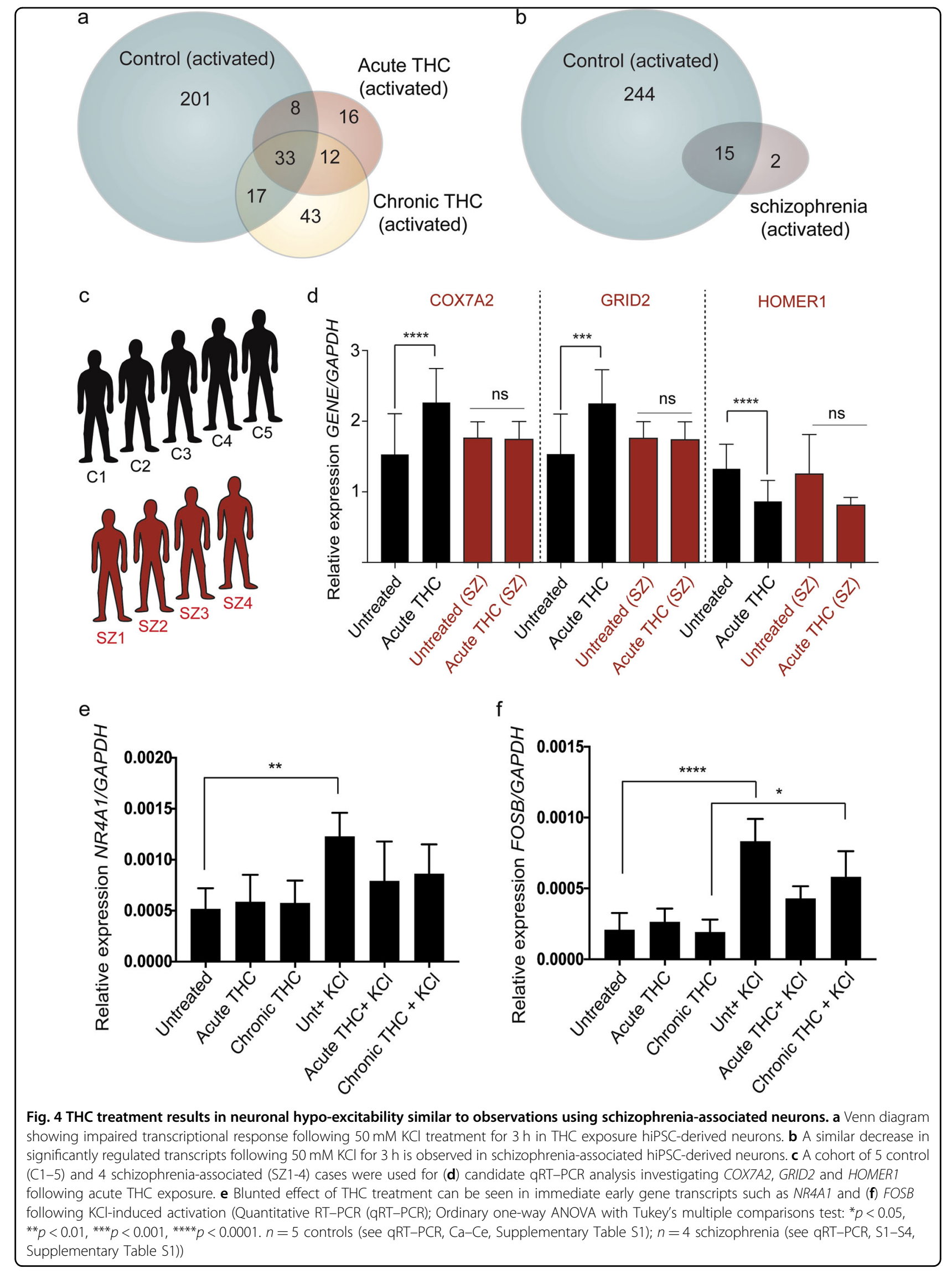


the enrichment of postsynaptic density genes in THC response (Fig. 2a).

Although we found abrogation of THC-induced changes in schizophrenia-associated hiPSC-derived neurons in candidate genes that were responsive to THC in our study, comprehensive biochemical and functional validation of our THC-induced effects are necessary, across both control and SZ neurons. Moreover, to confirm that these effects are mediated via cannabinoid signaling, future studies should attempt to recapitulate our observed effects using cannabinoid agonists (e.g., anandamide) or block them with selective antagonists (i.e., SR141716A). Consistent with this, we previously reported that THCinduced changes in gene expression in hiPSC neurons were blocked by concurrent $20 \mathrm{nM}$ SR141716A treatment ${ }^{23}$.

In summary, we found significant associations of THCrelated pathways to autism and intellectual disability. Furthermore, we have used a dynamic, human-relevant system to demonstrate a phenotypic link between THC treatment and schizophrenia. We hypothesize that THC exposure, by impacting many of the same synaptic and epigenetic pathways already associated with psychiatric disorders, may serve as an additive risk to existing genetic/ epigenetic risk factors.

\section{Acknowledgements}

K.J.B. is a New York Stem Cell Foundation-Robertson Investigator. The Brennand Laboratory is partially supported by a Brain and Behavior Young Investigator Grant, National Institute of Health (NIH) grant R01 MH101454 and the New York Stem Cell Foundation. The Roussos Laboratory is partially supported by the National Institutes of Health (R01AG050986 Roussos and R01MH109677 Roussos), Brain Behavior Research Foundation (20540 Roussos), Alzheimer's Association (NIRG- 340998 Roussos) and the Veterans Affairs (Merit grant BX002395 Roussos).

\section{Author contributions}

G.B., K.J.B. and Y.L.H. conceived of the experiments. K.J.B. generated the hiPSC neurons for RNA-seq while B.G. conducted the RNA-seq analyses guided by M. B., J.H. and P.R. D.C.K. performed RNA-seq experiments, E.A.O. conducted qPCR validation, while I.O. completed the control-SZ THC exposure experiments.

\begin{abstract}
Author details
'Sydney Medical School, Brain and Mind Centre, The University of Sydney, Camperdown, Sydney, NSW, Australia. ${ }^{2}$ St. Vincent's Clinical School and School of Biotechnology and Biomolecular Sciences, University of New South Wales, Kensington, NSW, Australia. ${ }^{3}$ Garvan Institute of Medical Research, Darlinghurst, NSW, Australia. ${ }^{4}$ QIMR Berghofer Medical Research Institute, Herston, QLD, Australia. ${ }^{5}$ Department of Psychiatry, Icahn School of Medicine at Mount Sinai, New York, NY, USA. ${ }^{6}$ Department of Neuroscience and Friedman Brain Institute, New York, NY, USA. ${ }^{7}$ Department of Genetics and Genomic Science and Institute for Multiscale Biology, New York, NY, USA. ${ }^{8}$ Mental Illness Research, Education, and Clinical Center (VISN 2), James J. Peters VA Medical Center, Bronx, NY, USA. ${ }^{9}$ Fishberg Department of Neuroscience, Icahn School of Medicine at Mount Sinai, New York, NY, USA
\end{abstract}

Conflict of interest

The authors declare that they have no conflict of interest.
Publisher's note

Springer Nature remains neutral with regard to jurisdictional claims in published maps and institutional affiliations.

Supplementary Information accompanies this paper at https://oi.org/ 10.1038/s41398-018-0137-3.

Received: 5 February 2018 Accepted: 18 February 2018

Published online: 25 April 2018

\section{References}

1. Boland, M. J. et al. Molecular analyses of neurogenic defects in a human pluripotent stem cell model of fragile X syndrome. Brain 140, 582-598 (2017).

2. Israel, M. A. et al. Probing sporadic and familial Alzheimer's disease using induced pluripotent stem cells. Nature 482, 216-220 (2012).

3. Ryan, S. D. et al. Isogenic human iPSC Parkinson's model shows nitrosative stress-induced dysfunction in MEF2-PGC1alpha transcription. Cell 155, 1351-1364 (2013).

4. Marchetto, M. C. et al. Altered proliferation and networks in neural cells derived from idiopathic autistic individuals. Mol. Psychiatry 22, 820-835 (2016).

5. Madison, J. M. et al. Characterization of bipolar disorder patient-specific induced pluripotent stem cells from a family reveals neurodevelopmental and mRNA expression abnormalities. Mol. Psychiatry 20, 703-717 (2015).

6. Roussos, P., Guennewig, B., Kaczorowski, D. C., Barry, G. \& Brennand, K. J. Activity-dependent changes in gene expression in schizophrenia humaninduced pluripotent stem cell neurons. JAMA Psychiatry 73, 1180-1188 (2016).

7. Weinstein, A., Livny, A. \& Weizman, A. Brain imaging studies on the cognitive, pharmacological and neurobiological effects of cannabis in humans: evidence from studies of adult users. Curr. Pharm. Des. 22, 6366-6379 (2016).

8. Colizzi, M., McGuire, P., Pertwee, R. G. \& Bhattacharyya, S. Effect of cannabis on glutamate signalling in the brain: A systematic review of human and animal evidence. Neurosci. Biobehav Rev. 64, 359-381 (2016).

9. Lu, H. C. \& Mackie, K. An introduction to the endogenous cannabinoid system. Biol. Psychiatry 79, 516-525 (2016).

10. Bossong, M. G. et al. Effects of delta9-tetrahydrocannabinol on human working memory function. Biol. Psychiatry 71, 693-699 (2012).

11. Sherif, M., Radhakrishnan, R., D'Souza, D. C. \& Ranganathan, M. Human laboratory studies on cannabinoids and psychosis. Biol. Psychiatry 79, 526-538 (2016).

12. Volk, D. W. \& Lewis, D. A. The role of endocannabinoid signaling in cortical inhibitory neuron dysfunction in schizophrenia. Biol. Psychiatry 79, 595-603 (2016).

13. Vaucher J., et al. Cannabis use and risk of schizophrenia: a Mendelian randomization study. Mol. Psychiatry 2017, https://doi.org/10.1038/mp.2016.252.

14. Gage, S. H., Hickman, M. \& Zammit, S. Association between cannabis and psychosis: epidemiologic evidence. Biol. Psychiatry 79, 549-556 (2016).

15. Ksir, C. \& Hart, C. L. Cannabis and psychosis: a critical overview of the relationship. Curr. Psychiatry Rep. 18, 12 (2016).

16. French, L. et al. Early cannabis use, polygenic risk score for schizophrenia and brain maturation in adolescence. JAMA Psychiatry 72, 1002-1011 (2015).

17. Topol, A. et al. Dysregulation of miRNA-9 in a subset of schizophrenia patientderived neural progenitor cells. Cell Rep. 15, 1024-1036 (2016).

18. Brennand, K. J. et al. Modelling schizophrenia using human induced pluripotent stem cells. Nature 473, 221-225 (2011).

19. Brennand, $K$ et al. Phenotypic differences in hiPSC NPCs derived from patients with schizophrenia. Mol. Psychiatry 20, 361-368 (2015).

20. Szutorisz, H., Egervari, G., Sperry, J., Carter, J. M. \& Hurd, Y. L. Cross-generational THC exposure alters the developmental sensitivity of ventral and dorsal striatal gene expression in male and female offspring. Neurotoxicol Teratol. 58, 107-114 (2016).

21. Hook, V. et al. Human iPSC neurons display activity-dependent neurotransmitter secretion: aberrant catecholamine levels in schizophrenia neurons. Stem Cell Rep. 3, 531-538 (2014).

22. Tortoriello, G. et al. Miswiring the brain: Delta9-tetrahydrocannabinol disrupts cortical development by inducing an SCG10/stathmin-2 degradation pathway. EMBO J. 33, 668-685 (2014)

23. Obiorah, M. H. IV, Stafford, K., Flaherty, E. K. \& Brennand, K. J. THC treatment alters glutamate receptor gene expression in human stem cell-derived neurons. Mol. Neuropsychiatry 3, 73-84 (2017). 
24. Singh, N., Hroudova, J. \& Fisar, Z. Cannabinoid-induced changes in the activity of electron transport chain complexes of brain mitochondria. J. Mol. Neurosci. 56, 926-931 (2015).

25. Athanasiou, A. et al. Cannabinoid receptor agonists are mitochondrial inhibitors: a unified hypothesis of how cannabinoids modulate mitochondrial function and induce cell death. Biochem. Biophys. Res. Commun. 364, 131-137 (2007).

26. Rajasekaran, A., Venkatasubramanian, G., Berk, M. \& Debnath, M. Mitochondrial dysfunction in schizophrenia: pathways, mechanisms and implications. Neurosci. Biobehav. Rev. 48, 10-21 (2015).

27. Stanslowsky, N. et al. Functional effects of cannabinoids during dopaminergic specification of human neural precursors derived from induced pluripotent stem cells. Addict. Biol. 22, 1329-1342 (2016).

28. Fisar, Z., Singh, N. \& Hroudova, J. Cannabinoid-induced changes in respiration of brain mitochondria. Toxicol. Lett. 231, 62-71 (2014).

29. Lipina, C., Inving, A. J. \& Hundal, H. S. Mitochondria: a possible nexus for the regulation of energy homeostasis by the endocannabinoid system? Am. J. Physiol. Endocrinol. Metab. 307, E1-E13 (2014).

30. Wolff, $V$. et al. Tetrahydrocannabinol induces brain mitochondrial respiratory chain dysfunction and increases oxidative stress: a potential mechanism involved in cannabis-related stroke. Biomed. Res. Int. 2015, 323706 (2015).

31. Goncalves, V. F., Andreazza, A. C. \& Kennedy, J. L. Mitochondrial dysfunction in schizophrenia: an evolutionary perspective. Hum. Genet. 134, 13-21 (2015).
32. Hunter, R. G. et al. Stress and corticosteroids regulate rat hippocampal mitochondrial DNA gene expression via the glucocorticoid receptor. Proc. Nat Acad. Sci. USA 113, 9099-9104 (2016).

33. Picard, M. et al. Mitochondrial functions modulate neuroendocrine, metabolic inflammatory, and transcriptional responses to acute psychological stress. Proc. Natl Acad. Sci. USA 112, E6614-E6623 (2015).

34. Acin-Perez, R. \& Enriquez, J. A. The function of the respiratory supercomplexes: the plasticity model. Biochim. Biophys. Acta 1837, 444-450 (2014).

35. Morales, P., Hurst, D. P. \& Reggio, P. H. Molecular targets of the phytocannabinoids: a complex picture. Prog. Chem. Org. Nat. Prod. 103, 103-131 (2017).

36. Voglis, G. \& Tavernarakis, N. The role of synaptic ion channels in synaptic plasticity. EMBO Rep. 7, 1104-1110 (2006).

37. Yasuda, T. \& Adams, D. J. Physiological roles of ion channels in adult neural stem cells and their progeny. J. Neurochem. 114, 946-959 (2010).

38. Chen, D., Yu, S. P. \& Wei, L. lon channels in regulation of neuronal regenerative activities. Transl. Stroke Res. 5, 156-162 (2014).

39. Stringer, S. et al. Genome-wide association study of lifetime cannabis use based on a large meta-analytic sample of 32330 subjects from the International Cannabis Consortium. Transl. Psychiatry 6, e769 (2016).

40. Sytnyk, V., Leshchyns'ka, I. \& Schachner, M. Neural cell adhesion molecules of the immunoglobulin superfamily regulate synapse formation, maintenance, and function. Trends Neurosci. 40, 295-308 (2017).

41. Fogel, A. I. et al. SynCAMs organize synapses through heterophilic adhesion. J. Neurosci. 27, 12516-12530 (2007). 